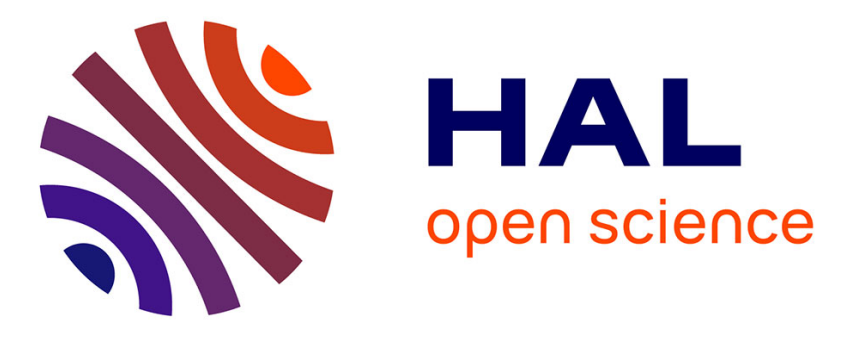

\title{
Longitudinal trajectories of emotions among young athletes involving in intense training centres: Do emotional intelligence and emotional regulation matter?
}

Valérian Cece, Emma Guillet-Descas, Virginie Nicaise, Noémie Lienhart, Guillaume Martinent

\section{To cite this version:}

Valérian Cece, Emma Guillet-Descas, Virginie Nicaise, Noémie Lienhart, Guillaume Martinent. Longitudinal trajectories of emotions among young athletes involving in intense training centres: Do emotional intelligence and emotional regulation matter?. Psychology of Sport and Exercise, 2019, 43, pp.128-136. 10.1016/j.psychsport.2019.01.011 . hal-02332137

\section{HAL Id: hal-02332137 \\ https://hal.science/hal-02332137}

Submitted on 22 Oct 2021

HAL is a multi-disciplinary open access archive for the deposit and dissemination of scientific research documents, whether they are published or not. The documents may come from teaching and research institutions in France or abroad, or from public or private research centers.
L'archive ouverte pluridisciplinaire HAL, est destinée au dépôt et à la diffusion de documents scientifiques de niveau recherche, publiés ou non, émanant des établissements d'enseignement et de recherche français ou étrangers, des laboratoires publics ou privés.

\section{(c) (1) $\$$}

Distributed under a Creative Commons Attribution - NonCommerciall 4.0 International 


\section{Running Head: LONGITUDINAL TRAJECTORIES OF SPORT EMOTIONS}

Longitudinal Trajectories of Emotions among Young Athletes Involving in Intense Training Centres: Do Emotional Intelligence and Emotional Regulation Matter?

Valérian Cece, Emma Guillet-Descas, Virginie Nicaise, Noémie Lienhart \& Guillaume Martinent

University of Claude Bernard Lyon 1 - University of Lyon, Laboratory of Vulnerabilities and Innovation in Sport (EA 7428), Interdisciplinary Confederation of Research in Sport (FED 4272), Lyon, France.

Date of submission: August 13, 2018

Date of revision 1: December 10, 2018

Date of revision 2: January 23, 2019

Address correspondence to Valérian Cece, University of Claude Bernard Lyon 1 University of Lyon, Laboratory of Vulnerabilities and Innovation in Sport, L-VIS (EA 7428), Interdisciplinary Confederation of Research in Sport (FED 4272), 27-29 Boulevard du 11 novembre, 69622 Villeurbanne, France. E-mail: valerian.cece@ orange.fr.

\section{Conflict of Interest}

All the authors declare that they have no conflict of interest. 
1 Running Head: LONGITUDINAL TRAJECTORIES OF SPORT EMOTIONS

2

3

$4 \quad$ Longitudinal Trajectories of Emotions among Young Athletes Involving in Intense Training

5 Centres: Do Emotional Intelligence and Emotional Regulation Matter?

6

7

8 Date of submission: August 13, 2018

9 Date of revision 1: December 10, 2018

10 Date of revision 2: January 23, 2019 


\begin{abstract}
\end{abstract}
Objectives. This study aimed to explore whether several subgroups of athletes representing distinct emotional trajectories emerged from the person-centred analyses for a wide variety of positive and negative sport emotions (anger, anxiety, sadness, confidence, happiness, harmony, love and vitality). The present study also focused on the determinants of the emotional dynamics exploring whether athletes reporting distinct scores of emotional intelligence (EI) and emotional regulation (ER) at time 1 (T1) belonged to distinct trajectories. Design. A longitudinal three-wave measurement design (beginning, middle, and end of a competitive season) was used in the present study. Method. A sample of 325 adolescent athletes evolving in intensive training centres completed three self-reported questionnaires. Results. Results of latent class growth analyses (LCGA) demonstrated four trajectories for love and confidence, three trajectories for anger, harmony, happiness, sadness, and vitality, and two trajectories for anxiety. Furthermore, the likelihood of belonging to emotional trajectories was significantly influenced by EI and ER scores for love, confidence, anger, harmony, happiness, sadness and vitality. Conclusions. LCGA results highlighted the young athlete heterogeneity in longitudinal sport emotions and the predictive effects of ER and EI on their emotional trajectories. These results could be used to help coaches and sport psychologists to identify athletes at risk of developing dysfunctional emotional trajectories across the competitive season. Moreover, this study suggested potential benefits of EI and ER-centred interventions to help young elite athletes to experience adaptive emotional trajectories. Finally, these results highlighted that the heterogeneity in sport emotions must be accounted for in future research.

Keywords: Emotional intelligence; Emotional regulation; Latent class growth analysis; Sport emotions; Young athletes. 
Longitudinal Trajectories of Emotions among Young Athletes Involving in Intense Training Centres: Do Emotional Intelligence and Emotional Regulation Matter?

There are a growing interest and an increase in the amount of research focusing on emotions in sport psychology (Campo, Mellalieu, Ferrand, Martinent, \& Rosnet, 2012; Cerin, Szabo, Hunt, \& Williams, 2000; Hanin, 2007). Emotions are central in a sport setting because of their significant influence on performance and well-being (Hanin, 2007; Lazarus, 2000; McCarthy, 2011). For instance, pleasant emotions (e.g. vitality, satisfaction) have been associated to positive outcomes such as mental health, performance or engagement (Lyubomirsky, King, \& Diener, 2005; Riemer \& Chelladurai, 1998) whereas unpleasant emotions such as anxiety, anger, and depression have been associated to negative consequences (e.g. athlete burnout) and/or positive consequences depending on the situation and/or individual (Beedie, Terry, \& Lane, 2000; Martinent, Ledos, Ferrand, Campo, \& Nicolas, 2015).

The Cognitive-Motivational-Relational theory (CMRT; Lazarus, 2000) postulates that the experience of discrete emotions depends on the singular way an athlete appraises the situation in which he is involved. In this perspective, emotion refers to an organized psychophysiological reaction (subjective experience, facial expression, physiological changes) to ongoing relationships with the environment reflecting the transaction between a person and his environment (Lazarus, 2000). Previous studies have suggested that the environment in which athletes are involved influences their emotional experiences (Campo et al., 2012). Hence, intensive training centres could influence the emotions experienced by young elite athletes because of the daily biological, social and psychological demands they have to cope with and/or the win-at-all-cost atmosphere that can reign in these centres (Martinent, Gareau, Lienhart, Nicaise, \& Guillet-Descas, 2018). In contrast, other authors have highlighted the facilitative role played by the sport competition context in the 
development of a wide variety of transferable skills for young elite athletes (Gaudreau, Amiot, \& Vallerand, 2009). Consequently, being involved in an intensive training centre is not an identical experience for all young elite athletes and different athletes could experience distinct emotional trajectories across the competitive season (Gaudreau et al., 2009; Lazarus, 2000; Martinent et al., 2018). Moreover, previous studies have provided evidence of the variability of emotional experiences across the competitive season as the daily constrains and competitive calendar can modulate athletes' emotional dynamics during the season (Cerin et al., 2000; Gaudreau et al., 2009; Martinent et al., 2018).

Although athletes experience a wide variety of pleasant and unpleasant discrete emotions in a sport setting (Hanin, 2007; Martinent, Campo, \& Ferrand, 2012), previous studies have historically focused on negative discrete emotions, especially on anxiety (Cerin et al. 2000). However, in line with the development of positive psychology (Lyubomirsky, King, \& Diener, 2005), recent researches have provided evidence for considering both positive and negative emotions because positive emotions do not simply refer to the absence of negative ones and vice versa (Folkman, 2013; Lundqvist \& Kenttä, 2010; McCarthy, 2011). Nevertheless, if some discrete emotions received considerable attention such as anxiety, anger, or happiness (Cerin, 2004; Cerin et al., 2000; McCarthy, 2011), other are currently poorly investigated in the sport context (e.g., love, harmony, vitality; Lundqvist \& Kenttä, 2010). Indeed, each discrete emotion could provide specific information (Lazarus, 2000) and complete knowledge about the emotional experience of individuals.

To take into account the dynamic and the heterogeneity of naturally-occurring emotional trajectories emerging across the competitive season, researchers have to use a methodological approach specifically designed to reveal subgroups of athletes belonging to particular emotional trajectories (Laursen \& Hoff, 2006). Longitudinal person-centred methods such as Latent Class Growth Analyses (LCGA) are suitable to identify several 
subgroups of athletes characterised by distinct emotional temporal patterns (or emotional trajectories) (Rindfleisch, Malter, Ganesan, \& Moorman, 2008). LCGA trajectories are characterized by an intercept, a linear slope and a quadratic slope. The intercept refers to the value at which a line cuts a coordinate axis which could be considered as low, moderate or high. The slopes define the dynamics of a selected-variable across the measurement times. The linear slope could be characterized as increasing (i.e. growing shape across the time), decreasing (i.e. declining shape across the time) or stable (i.e. no change across time) whereas the quadratic slope could be defined as positively unstable (i.e. U-shaped), negatively unstable (i.e. inverted U-shaped) or stable (i.e. no change across time). The examination of emotional trajectories across the competitive season could provide knowledge about the various emotional responses to the daily constraints of competitive atmosphere across the season. This approach could allow identifying some functional (i.e. theoretically associated to positive outcomes) or dysfunctional (i.e. theoretically associated to negative outcomes) subgroups into a global population. Thus, the emotional trajectories could be useful for both researchers and practitioners in providing further insights regarding the longitudinal everyday emotional experience. Yet only one study has already used such a methodology in sport psychology to examine longitudinal trajectories of positive and negative affective states with a sample of 265 adolescent elite hockey players followed across 3 measurement points during the first 11 weeks of a season (Gaudreau et al., 2009). LCGA results have provided evidence for 3 trajectories of positive affect (medium and decreasing, high and decreasing, unstable) and 3 trajectories of negative affect (low and unstable, medium and unstable, high and decreasing). Furthermore, athletes' memberships of trajectories of positive and negative affective states have been predicted by theoretically driven predictors assessed at the start of the season such as self-determination, need satisfaction, athletic identity, and school identity. These preliminary results have suggested that multinomial heterogeneity in longitudinal affective 
states needs to be accounted for in future research examining a wider range of unpleasant and pleasant discrete emotions (Gaudreau et al., 2009). Moreover, emotional trajectories of affective states have been based on a selected part of the competitive season (11 weeks) rather than on the whole competitive season.

In addition to identify distinct naturally-occurring emotional trajectories of a wide variety of unpleasant and pleasant emotions experienced by young athletes in intensive training centres, it could be particularly useful to explore the determinants of those emotional trajectories. In the present study, we focused on two individual-difference variables likely to predict membership of athletes' emotional trajectories across the competitive season: Emotional regulation (ER) and emotional intelligence (EI). ER refers to the process through which individuals influence which emotions they experience, when and how they experience and express them (Gross, 1998). Previous sport studies have shown that: (a) ER is linked to sport performance as well as to athletes' emotional experience (Jones, 2003; Martinent et al., 2015; Wagstaff, 2014); and (b) athletes use a wide variety of ER strategies to manage the emotions experienced in competition (Martinent et al., 2015) and during training (Nicholls, Holt, Polman, \& Bloomfield, 2006). Extending CMRT (Lazarus, 2000), the process model of emotion regulation (PMER) postulates that some ER strategies have an impact at different stages of the emotion generation process (Gross \& Thompson, 2007). PMER also helps to structure the variety of ER strategies distinguishing two ER families: Antecedent-focused and response-focused ER strategies. Antecedent-focused strategies act before the emotion response tendencies become fully activated (early in the emotion-generative process). One of the most investigated antecedent-focused ER strategies is reappraisal, which involves the positive reinterpretation of threatening situations or highlighting the positive consequences of emotion-eliciting situations (Gross \& John, 2003; John \& Gross, 2004). In line with CMRT, a positive reappraisal is supposed to positively change cognitions about the situation and may 
lead to high level of positive emotions and low level of negative ones (John \& Gross, 2004).

In contrast, response-focused strategies are aimed at modifying emotional response tendencies after they have already been triggered (Gross \& Thompson, 2007). One of the most investigated response-focused ER strategies is emotional suppression, which is designed to influence behavioral emotion responses by suppressing the expressive emotion response (Christophe, Antoine, Leroy, \& Delelis, 2009). Previous studies conducted in general (Gross \& Thomson, 2007) and sport psychology (Martinent et al., 2015; Wagstaff, 2014) have provided strong evidence that emotional suppression is accompanied by a heavy cognitive, physiological, and social cost compared with reappraisal. Thus, the frequent and steady repetition of this costly modulation of emotional responses could disturb the positive emotions or foster the experience of negative emotions (John \& Gross, 2004).

EI is another individual-difference variable examined in the present study which is linked to emotional experience (Mayer, 2009). EI refers to the abilities to identify, understand, regulate, and use one's and others' emotions (Davies, Lane, Devonport, \& Scott, 2010; Laborde, Dosseville, \& Allen, 2016; Schutte, Malouff, Simunek, McKenley, \& Hollander, 2002). A hierarchical structure of the concept of EI has been proposed, leading some researchers to use a global score of EI (Laborde et al., 2016). In particular, a bulk of studies have provided evidence that a global score of EI could be used by researchers and practitioners as they simultaneously encompass all the aforementioned dimensions of EI (Davies et al., 2010; Laborde et al., 2016). Based on the tenet of the CMRT (Lazarus, 2000), the level of global EI could impact the appraisal of a competitive situation. This modification of cognitive evaluation lead to positive or negative discrete emotions (Laborde et al., 2016). More precisely, athletes characterised by low scores of EI experienced high levels of anger and confusion whereas athletes characterised by high scores of EI reported low levels of anger, confusion, depression, fatigue and tension and high levels of calm and joy (Laborde et 
al. 2016). In the sport psychology literature, EI has been positively linked to athletes' pleasant emotion and sport performance and negatively linked to unpleasant emotions in many sports (Laborde et al., 2016). However, most of the previous studies have used a cross-sectional design, preventing researcher to clearly disentangle the temporal ordering between EI and emotions. Yet, in line with CMRT (Lazarus, 2000), we hypothesise that EI could predict the incoming positive and negative emotions.

In sum, emotional experiences are a crucial aspect of competitive sport especially for young elite athletes in intensive training centres as they are confronted with daily constraints. Considering that the emotional process is fluctuant across time (Lazarus, 2000) and that all young elite athletes do not necessarily experience the same emotions when involved in a demanding environment (Martinent et al., 2018), a LCGA approach (longitudinal personcentred method) seems suitable to examine longitudinal emotional trajectories of a wide variety of pleasant and unpleasant discrete emotions. Thus, in the present study, we explored whether several subgroups of athletes representing distinct emotional trajectories emerged from LCGAs for a wide variety of sport emotions (anger, anxiety, sadness, confidence, happiness, harmony, love and vitality). Furthermore, we examined whether athletes' dispositional ER and EI scores at the beginning of the season would predict membership of longitudinal emotional trajectories across the competitive season. Given that few studies examined the trajectories of various discrete emotions over the competitive season, no specific hypotheses were advanced regarding the number of trajectories, their characteristics (e.g., intercepts) or their evolution through time (e.g., linear and/or quadratic slopes). Concerning the relationships between ER, EI and longitudinal emotional trajectories, in line with the process model of emotion regulation (Gross, 1998; Gross \& Thompson, 2007), CMRT (Lazarus, 2000) and empirical research (Davies et al., 2010; Gross \& Jones, 2003; John \& Gross, 2004; Laborde et al., 2016; Martinent et al., 2015; Wagstaff, 2014), we 
hypothesised that: (a) young athletes reporting higher levels of dispositional EI and reappraisal would be more likely to belong to adaptive longitudinal emotional trajectories; and (b) athletes reporting higher levels of dispositional emotional suppression would be more likely to belong to maladaptive longitudinal emotional trajectories.

\section{Method}

\section{Participants}

A sample of three hundred and twenty-five youth athletes (122 females and 203 males, aged 16.06 years \pm 1.59 ) in intensive training centres participated in the study. Athletes practiced various team (basketball, soccer, rugby, handball, hockey, volleyball) and individual sports (judo, dance, track and field, swimming, boxing, cycling, golf, gymnastics). They participated in international $(\mathrm{n}=11)$, national $(\mathrm{n}=219)$ or regional $(\mathrm{n}=95)$ sport competitions. On average, they were invested in their sport for 8.12 years $( \pm 3.37)$ and trained $12.38 \mathrm{~h} /$ week $( \pm 3.33)$. Athlete's emotions scores were gathered in time 1 (T1) for 325 participants, in time 2 (T2) for 306 participants and in time 3 (T3) for 226 participants. The main reasons for attrition were disease, training camp, injury, or competition.

\section{Measures}

The Multiple Emotions Measure (MEM, Martinent et al., 2018) was used to measure emotions across the three measurement times. The MEM is a French questionnaire composed of eight 4-items subscales measuring anxiety, love, confidence, anger, harmony, happiness, sadness and vitality (Cronbach's $\alpha$ ranged from .76 to .87 for Martinent et al., 2018 and ranged from .74 to .92 for the present study). Participants responded on a 5-point Likert scale with values ranging from 1 (almost never) to 5 (most of the time).

The French version of the Brief Emotional Intelligence Scale (BEIS; Internal consistencies provided by Davies et al., 2010: $X^{2}=180.78, p<.05$, Comparative Fit Index $=$ .91 , Root Mean Square Error of Approximation $=.06$ ) was used to assess EI at the beginning 
211 of the season. This scale, derived from the Emotional Intelligence Scale (EIS; Schutte et al.,

212 1998), is a short and efficient measure of trait-EI (Davies et al., 2010). This questionnaire

213 provides a global score of EI ( $\alpha=.66$ for the present study) using 10 items focusing on 214 appraisal of own emotions, appraisal of others' emotions, regulation of own emotions, 215 regulation of others' emotions, and utilization of emotions. Participants responded on a 7216 point Likert type scale ranging from 1 (not at all agree) to 7 (completely agree).

217 The French version (Internal consistencies provided by Christophe et al., 2009: $X^{2}=$ 218 180.78, $p<.05$, Comparative Fit Index $=.90$, Root Mean Square Error of Approximation $=$ 219.05 ) of the Emotional Regulation Questionnaire (ERQ, Gross \& John, 2003) was used to 220 measure dispositional reappraisal $(\alpha=.79$ for Gross $\&$ John, $2003 ; \alpha=.83$ for the present study; 6 items) and emotional suppression ( $\alpha=.73$ for Gross \& John, 2003; $\alpha=.72$ for the present study; 4 items). Participants responded on a 7-point Likert type scale ranging from 1

223 (not at all agree) to 7 (completely agree).

\section{Procedure}

The present study was conducted according to the international ethical guidelines. The heads of high school and the coaches of the training centres comprised in the study were contacted before the data collection. The athletes' participation was voluntary. A written informed consent was obtained before the beginning of the study. Their parents also informed their contentment. Athletes completed the questionnaires during school courses or in their usual training place. The BEIS and ERQ were completed only at T1 whereas we processed to three measurement times for the MEM (T1, T2, and T3).

\section{Data analyses}

LCGAs were conducted on MPlus Version 7.3 (Los Angeles, CA, USA). We used the full information maximum likelihood (FIML) estimation of missing data which is an unbiased and more efficient method under the missing at random assumption than listwise deletion, 
which could lead to biased parameters (Enders, 2010). LCGA is a statistical model which posits that an underlying grouping variable can be inferred from a set of indicators to discover distinct trajectories on a psychological variable with different patterns of change and stability (Louvet, Gaudreau, Menaut, Genty, \& Deneuve, 2009; Martinent, Louvet, \& Decret, 2016). As longitudinal factor invariance is a prerequisite when conducting such analyses, preliminary analyses provided evidence for the strict temporal longitudinal invariance of MEM scores, signifying that changes in emotion reflected the real changes of the athletes' emotions over time without a bias interpretation due to temporal non-invariance.

Eight sets of analyses were performed, one for each discrete emotion. First, we conducted a series of unconditional LCGA models to select a model that precisely captured the shape and the number of the trajectories describing each of the emotions). Thus, a succession of models with increasing number of trajectories was achieved to identify which model was associated with the best-fit indices (Jung \& Wickrama, 2008). We used a mixture of statistical indices to determine the best-fitting model making up the log-likelihood value, the Akaike Information Criterion (AIC), the Bayesian Information Criterion (BIC), the Adjusted BIC (ABIC), and the Lo, Mendell, and Rubin likelihood ratio test (LRT). The highest log-likelihood scores and the smallest values of AIC, BIC, and ABIC designated the best-fitting model. Initial LCGA models included the mean level (intercept) and the linear and quadratic growths for each trajectory. Then LCGA models with both linear and quadratic functions were compared with their respective LCGA models with only the linear function. The log likelihood ratio test allowed highlighting an eventual significant improvement of fit if fewer parameters were included in the model (i.e., omitting quadratic functions from LCGA models). Second, we examined whether EI and ER measured at T1 (i.e. beginning of the season) could predict membership of emotional trajectories. Thus, EI and ER scores were included as covariates of the selected models using the three-step model (Nylund-Gibson, 
261 Grimm, Quirk, \& Furlong, 2014). This approach fixes model parameters (intercept, linear

262 and/or quadratic slopes) of the several longitudinal emotional trajectories at the values from

263 the unconditional LCGA models. In other words, three-step models permitted to include the

264 covariates in the mixture models without that covariates alter class estimation (Asparouhov \&

265 Muthén, 2014).

266

\section{Results}

267

268

269

270

271

272

273

274

275

276

277

278

279

280

281

282

283

284

285

\section{Unconditional LCGA Models}

Results of unconditional LCGAs are presented in Table 1. For love and confidence, we observed big drops of AIC, BIC, and ABIC between 3 and 4 classes. Additionally, LRT revealed that 4 classes fit better than 3 classes while 5 classes did not fit better than 4 classes for introjected regulation. When comparing LCGA models, it is important to consider not only the statistical indicators but also the substantive meaning of each of the emerging trajectories when interpreting the LCGA results (Martinent et al., 2016). Thus, to achieve the balance between theoretical and statistical considerations, we used the model parameters to make sense of the classes and decide which model fits best. Based on the interpretability of the emotional trajectories and statistical indicators, we selected a four-class solution for love and confidence. For anger, harmony, happiness, sadness and vitality, the AIC, BIC, ABIC, and LRT indicated that the 3-class models fit best. Specifically, there were big drops between 2 and 3 classes for the AIC, BIC and ABIC. LRT also revealed that 3 classes fit better than 2 classes while 4 classes did not fit better than 3 classes. Based on the interpretability of the emotional trajectories (the three-class solutions made theoretical sense whereas a fourth trajectory did not add anything substantive to the understanding of emotional trajectories) and the LCGA statistical indicators, a three-class solution was selected for anger, harmony, happiness, sadness, and vitality. For the anxiety, there were big drops of AIC, BIC, and ABIC between 1 and 2 classes. Additionally, LRT revealed that 2 classes fit better than 1 class while 
3 classes did not fit better than 2 classes for anxiety. A two-class solution was selected for anxiety.

Moreover, it is worth noting that the LRTs indicated significant worsening of fit if quadratic functions were omitted for love $(\mathrm{LRT}=27.40, \Delta \mathrm{df}=4, p<.001)$, anxiety (LRT = $5.68, \Delta \mathrm{df}=2, p=.05)$, anger $(\mathrm{LRT}=6.80, \Delta \mathrm{df}=3, p=.05)$, happiness $(\mathrm{LRT}=13.41, \Delta \mathrm{df}=$ $3, p<.005)$ and sadness (LRT $=12.37, \Delta \mathrm{df}=3, p<.01)$. Thus, both the linear and quadratic parameters were selected for these emotions. However, for confidence, harmony, and vitality $(\mathrm{LRT}=6.59,3.50$, and 5.40 respectively, $\Delta \mathrm{df}=3$ or $4, p>.10)$, the LRT indicated nonsignificant worsening of fit if quadratic functions were omitted. Thus, the linear parameters were only selected for these emotions.

\section{Conditional LCGA Models with Covariates}

\section{Interpreting the Emotional Trajectories}

The trajectories' estimates for the conditioned LCGA models are presented in Table 2. For love, the high-and-stable $(n=181)$ and moderate-and-stable $(n=9)$ subgroups represented athletes who always experienced high $($ intercept $=3.86, p<.01$, linear $=-.08, p=$ .49 , quadratic $=.06, p=.21)$ and moderate (intercept $=3.46, p<.01$, linear $=-.67, p=.39$, quadratic $=-.02, p=.96$ ) levels of love across the three time points. The high-and-unstable group $(n=33)$ referred to high levels of love, a marginally positive linear function and a negative quadratic function (intercept $=3.77, p<.01$, linear $=.39, p=.08$, quadratic $=-.37, p$ $<.01)$ whereas the very-high-and-unstable $(n=102)$ subgroup described athletes who experienced very high level of love and a positive quadratic function (intercept $=4.47, p<$ .01 linear $=-.15, p=.20$, quadratic $=.17, p<.01)$. For confidence, the low-and-increasing $(n$ $=52)$, the moderate-and-increasing $(n=11)$ and the high-and-increasing $(n=89)$ subgroups represented athletes who experienced low (intercept $=2.24, p<.01$, linear $=.19, p=.05$ ), moderate $($ intercept $=2.61, p<.01$, linear $=1.06, p<.01)$ and high $($ intercept $=4.37, p<$ 
.01 , linear $=.15, p=.02$ ) level of confidence (respectively) and a positive linear function. The high-and-decreasing $(n=173)$ subgroup referred to high level of confidence and a marginally negative linear function (intercept $=3.62, p<.01$, linear $=-.93, p=.08$ ). For harmony, the high-and-stable $(n=145)$, moderate-and-stable $(n=166)$ and low-and-stable $(n=14)$ subgroups represented athletes who always experienced high (intercept $=4.30, p<.01$, linear $=-.01, p=.85)$, moderate (intercept $=3.29, p<.01$, linear $=.05, p=.32)$, and low (intercept $=1.88, p<.01$, linear $=.20, p=.13)$ levels of harmony across the measurement times. For happiness, the very-high-and-decreasing $(n=101)$ subgroup referred to very high level of happiness and a negative linear function (intercept $=4.69, p<.01$, linear $=-.43, p=.01$, quadratic $=.13, p=.13)$ whereas the moderate-and-increasing $(n=40)$ subgroup represented athletes who experienced moderate level of happiness and a positive linear function (intercept $=2.66, p<.01$, linear $=.64, p=.03$, quadratic $=-.15, p=.30$. The high-and-stable $(n=180)$ subgroup represented athletes who always experienced high levels of happiness (intercept $=$ $3.72, p<.01$, linear $=-.13, p=.26$, quadratic $=.07, p=.25)$. For vitality, the high-and-stable $(n=133)$ subgroup represented athletes who always experienced high level of vitality (intercept $=4.66, p<.01$, linear $=-.06, \mathrm{p}=.44$ ) across the season whereas the low-andincreasing $(n=27)$ subgroup referred to low level of vitality and a positive linear function (intercept $=2.75, p<.01$, linear $=.40, p=.05)$. The moderate-and-decreasing $(n=165)$ subgroup described athletes who experienced moderate level of vitality and a marginally negative linear function $($ intercept $=3.88, p<.01$, linear $=-.11, p=.07$ ).

For anxiety, the moderate-and-unstable $(n=130)$ subgroup represented athletes who experienced moderate level of anxiety, a marginally positive linear function and a marginally negative quadratic function (intercept $=3.08, p<.01$, linear $=.42, p=.06$, quadratic $=-.19, p$ $=.09)$ whereas the low-and-stable $(n=195)$ subgroup referred to low level of anxiety across the three measurement times (intercept $=1.88, p<.01$, linear $=.12, p=.49$, quadratic $=-.09$, 
$p=.28)$. For anger, the moderate-and-stable $(n=93)$ subgroup represented athletes who

always experienced moderate level of anger across the season (intercept $=2.60, p<.01$, linear $=-.20, p=.59$, quadratic $=.10, p=.55)$. The high-and-decreasing $(n=22)$ subgroup described athletes who experienced high level of anger and a marginally negative linear function $($ intercept $=3.87, p<.01$, linear $=-.88, p=.07$, quadratic $=.27, p=.27$ ) whereas the low-and-unstable $(\mathrm{n}=210)$ subgroup referred to low level of anger, a positive linear function and a negative quadratic function (intercept $=1.37, p<.01$, linear $=.47, p<.01$, quadratic $=$ $.18, p=.01)$. For sadness, the low-and-stable $(n=241)$ subgroup represented athletes who always experienced low level of sadness (intercept $=1.33, p<.01$, linear $=.11, p=.44$, quadratic $=-.03, p=.72$. The high-and-unstable $(n=33)$ subgroup referred to high level of sadness, a negative linear function and a positive quadratic function (intercept $=3.80, p<.01$, linear $=-1.80, p<.01$, quadratic $=.68, p<.01)$ whereas the low-and-unstable $(n=51)$ subgroup described athletes who experienced low level of sadness, a positive linear function and a negative linear function (intercept $=1.77, p<.01$, linear $=2.32, p<.01$, quadratic $=-$ $.93, p<.01)$.

\section{Effect of Emotional Intelligence and Emotional Regulation on Membership of}

\section{Emotional Trajectories}

The logistic regression coefficients highlighting the effects of EI and ER on memberships of emotional trajectories are presented in Table $3^{1}$. The likelihoods of belonging to the high-and-stable and high-and-unstable trajectories of love - relative to the very-highand-unstable trajectory of love - were negatively related to T1 EI (ORs $=.33$ and .28 respectively, $p_{s}=.02$ ). The likelihoods of belonging to the high-and-decreasing and low-andincreasing trajectories of confidence - relative to the high-and-increasing trajectory of

\footnotetext{
${ }^{1}$ We also computed a series of LCGA model in which EI, reappraisal, emotional suppression, and the interaction terms of El x reappraisal and El $x$ emotional suppression were simultaneously included as predictors of emotional trajectories. Results revealed no significant results for the interaction terms. Therefore, the interaction terms were not included within the final conditional LCGA models.
} 
confidence - were negatively related to $\mathrm{T} 1 \mathrm{EI}\left(\mathrm{ORs}=.26\right.$ and $.38, p_{s}=.02$ and .06$)$; the likelihoods of belonging to the low-and-increasing trajectory of confidence - relative to the high-and-increasing trajectory of confidence - were negatively related to T1 reappraisal (OR $=.64, p=.04$ ). The likelihoods of belonging to the moderate-and-stable trajectory of harmony - relative to the high-and-stable trajectory of harmony - were negatively related to T1 reappraisal $(\mathrm{OR}=.61, p=.02)$ and to $\mathrm{T} 1 \mathrm{EI}(\mathrm{OR}=.14, p<.01)$. The likelihoods of belonging to the low-and-stable trajectory of harmony - relative to the high-and-stable trajectory of harmony - were negatively related to $\mathrm{T} 1$ reappraisal and marginally related to $\mathrm{T} 1$ emotional suppression $\left(\mathrm{ORs}=.45\right.$ and $1.59, p_{s}=.00$ and .08$)$. The likelihoods of belonging to the moderate-and-increasing trajectory of happiness - relative to the very-high-and-decreasing trajectory of happiness - were negatively related to $\mathrm{T} 1$ reappraisal and to $\mathrm{T} 1 \mathrm{EI}(\mathrm{ORs}=.37$ and $\left..13, p_{s}<.01\right)$ and positively related to $\mathrm{T} 1$ emotional suppression $(\mathrm{OR}=.1 .73, p=.01)$. The likelihoods of belonging to the high-and-stable trajectory of happiness - relative to the very-high-and-decreasing trajectory of happiness - were negatively related to T1 EI $(\mathrm{OR}=$ $.18, p<.01)$ and positively related to $\mathrm{T} 1$ emotional suppression $(\mathrm{OR}=.1 .41, p=.04)$. The likelihoods of belonging to the low-and-increasing trajectory of vitality - relative to the highand-stable trajectory of vitality - were negatively related to $\mathrm{T} 1$ reappraisal and $\mathrm{T} 1 \mathrm{EI}(\mathrm{ORs}=$ .53 and $\left..26, p_{s}=.01\right)$. The likelihoods of belonging to the moderate-and-decreasing trajectory of vitality - relative to the high-and-stable trajectory of vitality - were negatively related to $\mathrm{T} 1$ reappraisal $(\mathrm{OR}=.65, p=.01)$ and positively related to $\mathrm{T} 1$ suppression $(\mathrm{OR}=1.38, p=$ $.04)$.

The likelihoods of belonging to the low-and-unstable trajectory of anger - relative to the high-and-decreasing trajectory of anger - were positively related to $\mathrm{T} 1$ reappraisal $(\mathrm{OR}=$ $1.61, p=.03)$. The likelihoods of belonging to the low-and-stable trajectory of sadness relative to the high-and-unstable trajectory of sadness - were negatively related to T1 
emotional suppression $(\mathrm{OR}=1.49, p=.02)$ and positively related to $\mathrm{T} 1$ reappraisal $(\mathrm{OR}=$ $.70, p=.02$ ). The likelihoods of belonging to the low-and-unstable trajectory of sadness relative to the high-and-unstable trajectory of sadness - were positively related to $\mathrm{T} 1$ reappraisal $(\mathrm{OR}=1.68, p=.03)$.

\section{Discussion}

The aim of the present study was to examine whether several subgroups of athletes representing distinct longitudinal emotional trajectories emerged from LCGAs. A wide range of discrete emotions (anger, anxiety, sadness, confidence, happiness, harmony, love, and vitality) were investigated for a sample of young elite athletes involved in intensive training settings followed across 3 measurement points during the competitive season (beginning, middle and end of the sport season). We also explored whether dispositional ER and EI scores reported by youth athletes at the beginning of the season would predict membership of emerging longitudinal emotional trajectories across the competitive season. Results of LCGAs revealed several distinct emotional trajectories for each of the eight discrete emotions. In particular, four trajectories for love (very high and unstable, high and stable, high and unstable, moderate and stable) and confidence (high and increasing, high and decreasing, moderate and increasing, low and increasing), three trajectories for anger (high and decreasing, moderate and stable, low and unstable), harmony (high and stable, moderate and stable, low and stable), happiness (very high and decreasing, high and stable, moderate and increasing), sadness (high and unstable, low and stable, low and unstable) and vitality (high and stable, moderate and decreasing, low and increasing) and two trajectories for anxiety (moderate and unstable, low and stable) emerged from the LCGAs. Thus, the results revealed that each discrete emotion is characterised by a specific degree of interindividual variability. For instance, in the present study, confidence experiences are particularly heterogeneous as four classes emerged from the LCGAs whereas anxiety experiences could be captured with 
only two subgroups. As a whole, these results provided evidence for the usefulness of exploring several discrete emotions as distinct trajectories emerging for each of the eight discrete emotions across the competitive season. Indeed, in line with CMRT, results of this study suggested that each specific emotion "tell(s) a different story about a person's adaptational struggle" (Lazarus, 2000, pp. 232).

Confirming the results of Gaudreau et al.' (2009) study focusing on positive and negative affective states, those of the present study demonstrated that it is useful to take into account heterogeneity in longitudinal sport emotion across the competitive season for a wide range of pleasant and unpleasant discrete emotions. Indeed, naturally-occurring emotional regulation strategies emerging from the LCGAs highlighted both change (significant linear and/or quadratic slopes) and stability at an intra-individual level of analysis for most of the discrete emotions examined. Albeit with a different methodology, these results are consistent with previous sport studies depicting a nomothetic-idiographic process for the construct of emotion (Cerin, 2004; Martinent et al., 2012, 2018). Specifically, it is important to outline that some but not all athletes have exhibited changes in their experience of discrete emotions across time. For instance, several athletes experienced stable discrete emotions across the three measurement times (beginning, middle, and end of the season) $-74 \%$ of athletes belonged to stable trajectories for sadness, $60 \%$ for anxiety, $58 \%$ for love, $55 \%$ for happiness, $41 \%$ for vitality, and $29 \%$ for anger. These athletes do not seem really affected by the intensive training environment and influenced by the competitive or personal events during the season. For harmony, all athletes belonged to stable emotional trajectories (high and stable, moderate and stable, or low and stable). Thus, we could considerate that this discrete emotion has a little temporal variability during a competitive season. In contrast, all the athletes exhibited changes of confidence across the season as the four emerging trajectories for confidence were characterized by linear and/or quadratic changes across time (high and 
increasing, high and decreasing, moderate and increasing, low and increasing). These changes

could refer to the consequences of the athletes' everyday life (Martinent et al., 2018). Indeed, the individual response to the positive and negative competitive events or personal experiences could lead to an increase or a decrease in emotional scores across the season. For instance, the decreasing of positive emotions could be explained by the daily pressure and the biological, psychological and social demands (Gaudreau et al., 2009).

Based on a theoretical background such as the broaden and built theory (Fredrickson, 2001) and on previous studies in sport psychology (McCarthy, 2011; Riemer \& Chelladurai, 1998), some trajectories could be characterized as maladaptive (i.e. dysfunctional) whereas other could be considered as adaptive (i.e. functional). Because low scores of dispositional unpleasant emotions and high scores of dispositional pleasant emotions have been associated with various positive outcomes (e.g., well-being, performance) and/or an absence of negative ones (e.g., burnout) (Beedie et al., 2000; Campo et al., 2012; Martinent et al., 2016; McCarthy, 2011), it seems reasonable to postulate that the high-and-stable trajectories of love, harmony, vitality and happiness, the moderate-and-increasing and high-and-increasing trajectories of confidence, and the low-and-stable trajectories of anxiety and sadness could be conceptualized as functional emotional trajectories. In contrast, the moderate-and-decreasing trajectory of vitality, low-and-stable trajectory of harmony, moderate-and-unstable trajectory of anxiety and high and unstable trajectory of sadness could be conceptualized as dysfunctional emotional trajectories. For other emotional trajectories (e.g., high-anddecreasing trajectory of anger or low-and-increasing trajectory of confidence), it seems more difficult to definitely characterize these trajectories as functional versus dysfunctional ones. To unambiguously characterize such emerging emotional trajectories, upcoming research could explore the effect of memberships of emotional trajectories on several distal outcomes (e.g. performance, well-being, burnout) measured at the end of the season or several years 
later. Nevertheless, the influence of dispositional ER and EI scores assessed at the beginning of the season on membership of emotional trajectories across the competitive season could provide preliminary information about the functional versus dysfunctional nature of emotional trajectories in the context of the present study.

Results of LCGAs revealed that dispositional EI and ER scores at T1 significantly predicted membership of emotional trajectories for love, anger, confidence, harmony, happiness, sadness and vitality. In particular, young elite athletes reporting higher levels of dispositional EI were more likely belonging to functional longitudinal emotional trajectories compared with less functional longitudinal emotional trajectories. These results are in line with previous studies focused on EI (Davies et al., 2010; Laborde et al., 2016) confirming the positive influence of EI on emotions. Indeed, athletes characterised by high scores of EI at the beginning of the season may use their abilities to overcome the daily constraints of the competitive season. Specifically, in line with CMRT (Lazarus, 2000), a better identification, understanding, regulation and use of emotions may modulate their appraisal of the competitive experiences. These capacities to modify the cognitive evaluation of sport situations may enable athletes to experience functional emotional trajectories during the season. In the same way, athletes reporting higher scores of reappraisal were also more likely belonging to functional longitudinal emotional trajectories confirming previous studies focused on ER (John \& Gross, 2004; Martinent et al., 2015). The young athletes who used reappraisal strategies at the beginning of the season may positively change their cognitions about the competitive situations. Thus, the ability to use positive reappraisal strategies seems to promote functional emotional trajectories across the season. In contrast, young elite athletes reporting higher levels of dispositional emotional suppression at T1 were more likely belonging to dysfunctional longitudinal emotional trajectories compared with less dysfunctional emotional trajectories. These results are in line with previous studies 
highlighting the high cognitive, physiological and social cost of emotional regulation compared with reappraisal (John \& Gross, Wagstaff, 2014). Indeed, the recurrence of suppression strategies may disturb the emotional experiences during the season and lead to dysfunctional emotional trajectories. Overall, the results of LCGA are in line with the CMRT confirming the role of EI and ER on cognitive appraisal and emotional experiences in a sport setting (Davies et al., 2010; John \& Gross, 2004; Laborde et al., 2016; Martinent et al., 2015). Moreover, the present results highlight the temporal effect of EI and ER at the beginning of the season on emotional trajectories across a competitive season.

These results could be used to help coaches and sport psychologists working with adolescent athletes involved in intensive training centres. The methodological approach used in this study could be useful to identify dysfunctional emotional trajectories and ultimately to help the creation of targeted and adapted intervention to subgroups of young athletes involved in intensive training centres. Knowing which form of ER (reappraisal versus emotional suppression) and whether EI is related to which emotional trajectory is crucial to the development of well-designed prevention and intervention program. Based on the premise that emotional experience can be seen as a nomothetic-idiographic process (Cerin, 2004; Martinent et al., 2018), sport psychologists could try to develop empirically-proven interventions to help young athletes change their dysfunctional emotional trajectories and/or stabilize their functional emotional trajectories over time to maximize their psychological adjustment to the high demands inherent to the environment of intensive training centres. Based on the LCGA results, interventions should be targeted toward young athletes reporting low dispositional EI and reappraisal and/or high dispositional emotional suppression because these young athletes were found to be the more at risk of developing dysfunctional emotional trajectories across the competitive season. Based on the tripartite model of EI (Mikolajczak, 2009) and on previous empirical studies highlighting that it was possible to increase 
dispositional EI scores (Nelis, Quoidbach, Mikolajczak, \& Hansenne, 2009), sport psychologists could implement specific programs designed to enhance young athletes' dispositional EI (e.g., increasing knowledge about emotions and emotion regulation, using pre-competitive and intra-competitive routines; Campo, Laborde, \& Weckemann, 2015).

The present research has some limitations that should be acknowledged. First, before generalizing the findings, caution should be taken given the specificity of our population (young athletes in intensive training settings). Second, the absence of distal outcomes prevented us to unambiguously examine the functional versus dysfunctional nature of the emerging emotional trajectories. Consequently, future studies should include several outcomes variables in the study design, such as performance or well-being indicators. Third, although a longitudinal design based on the competitive season was used in the present study, sport carrier is longer than this time period. Indeed, the succession of competitive seasons could help to describe other emotional trajectories. In this perspective, future studies should extend the knowledge base about the longitudinal development of emotions in the sport context in focusing on four competitive seasons (representing an Olympiad). Moreover, it is noteworthy that EI and ER represent particular predictive factors in a multifactorial system (e.g. motivation, training history) and it could be useful to explore the influence of other factors in future studies to provide further insights on emotional experience in a sport context.

Despite these limitations, the present study furthered our knowledge base on sport emotions by providing new insights on longitudinal everyday experience of eight discrete emotions (love, anxiety, anger, confidence, harmony, happiness, sadness and vitality) with a sample of young elite athletes involved in intensive training centres followed across 3 measurement points during the competitive season (beginning, middle and end of the sport season). The person-centred method used in the present study highlighted that the heterogeneity in longitudinal sport emotions needs to be accounted for to identify intra- 
534 individual emotional trajectories experienced by athletes through the competitive season.

535 Moreover, results of LCGAs also demonstrated that dispositional EI and ER assessed at the

536 beginning of the season significantly predicted membership of longitudinal emotional

537 trajectories across the competitive season. In particular, EI and reappraisal predicted

538 belonging to functional emotional trajectories whereas emotional suppression predicted

539 belonging to dysfunctional emotional trajectories. These findings are interesting for sport

540 psychology because they help to target particular athletes at risk of developing dysfunctional

541 emotional trajectories across the competitive season.

542

543

544

545

546

547

548

549

550

551

552

553

554

555

556

557

\section{Conflicts of interest}

All the authors declare that they have no conflict of interest.

\section{Funding}

This research did not receive any specific grant from funding agencies.

\section{References}

Asparouhov, T., \& Muthén, B. (2014). Auxiliary variables in mixture modeling: Three-step approaches using M plus. Structural Equation Modeling: A Multidisciplinary Journal, 21(3), 329-341. https://doi.org/10.1080/10705511.2014.915181

Beedie, C. J., Terry, P. C., \& Lane, A. M. (2000). The profile of mood states and athletic performance: Two meta-analyses. Journal of Applied Sport Psychology, 12(1), 49-68. https://doi.org/10.1080/10413200008404213

Campo, M., Laborde, S., \& Weckemann, S. (2015). Emotional intelligence training: Implications for performance and health. In A. M. Columbus (Ed.), Advances in psychology research (pp. 75-92). Hauppauge, NY: Nova Science Publishers.

Campo, M., Mellalieu, S., Ferrand, C., Martinent, G., \& Rosnet, E. (2012). Emotions in team contact sports: A systematic review. The Sport Psychologist, 26(1), 62-97. 
Cerin, E. (2004). Predictors of competitive anxiety direction in male Tae Kwon Do practitioners: A multilevel mixed idiographic/nomothetic interactional approach. Psychology of Sport and Exercise, 5(4), 497-516. https://doi.org/10.1016/S14690292(03)00041-4

Cerin, E., Szabo, A., Hunt, N., \& Williams, C. (2000). Temporal patterning of competitive emotions: A critical review. Journal of Sports Sciences, 18(8), 605-626. https://doi.org/10.1080/02640410050082314

Christophe, V., Antoine, P., Leroy, T., \& Delelis, G. (2009). Évaluation de deux stratégies de régulation émotionnelle: la suppression expressive et la réévaluation cognitive. European Review of Applied Psychology, 59(1), 59-67. https://doi.org/10.1016/j.erap.2008.07.001

Davies, K. A., Lane, A. M., Devonport, T. J., \& Scott, J. A. (2010). Validity and reliability of a brief emotional intelligence scale (BEIS-10). Journal of Individual Differences. https://doi.org/10.1027/1614-0001/a000028

Enders, C. K. (2010). Applied missing data analysis. Guilford Press.

Folkman, S. (2013). Stress: appraisal and coping. In M. D. Gellman \& J. R. Turner (Éd.), Encyclopedia of behavioral medicine (p. 1913-1915). NY: Springer.

Fredrickson, B. L. (2001). The role of positive emotions in positive psychology: The broadenand-build theory of positive emotions. American Psychologist, 56, 218-226. doi:10.1037//0003-066X.56.3.218

Gaudreau, P., Amiot, C. E., \& Vallerand, R. J. (2009). Trajectories of affective states in adolescent hockey players: Turning point and motivational antecedents. Developmental Psychology, 45(2), 307. https://doi.org/10.1037/a0014134

Gross, J. J. (1998). The emerging field of emotion regulation: an integrative review. Review of General Psychology, 2(3), 271. https://doi.org/10.1037/1089-2680.2.3.271 
Gross, J. J., \& John, O. P. (2003). Individual differences in two emotion regulation processes: implications for affect, relationships, and well-being. Journal of Personality and Social Psychology, 85(2), 348. https://doi.org/10.1037/0022-3514.85.2.348

Gross, J. J., \& Thompson, R. A. (2007). Emotion regulation: Conceptual foundations. In J. J. Gross (Ed.), Handbook of emotion regulation (pp. 3-24). New York, NY: Guilford

Hanin, Y. L. (2007). Emotions in sport: Current issues and perspectives. In G. Tenenbaum \& R. C. Eklund (Éd.), Handbook of Sport Psychology (Vol. 3, p. 31-58). Hoboken: NJ: John Wiley \& Sons.

John, O. P., \& Gross, J. J. (2004). Healthy and unhealthy emotion regulation: Personality processes, individual differences, and life span development. Journal of Personality, 72(6), 1301-1334. https://doi.org/10.1111/j.1467-6494.2004.00298.x

Jones, M. (2003). Controlling emotions in sport. The Sport Psychologist, 17(4), 471-486. https://doi.org/10.1123/tsp.17.4.471

Jung, T., \& Wickrama, K. A. S. (2008). An introduction to latent class growth analysis and growth mixture modeling. Social and Personality Psychology Compass, 2(1), 302317. https://doi.org/10.1111/j.1751-9004.2007.00054.x

Laborde, S., Dosseville, F., \& Allen, M. S. (2016). Emotional intelligence in sport and exercise: A systematic review. Scandinavian Journal of Medicine \& Science in Sports, 26(8), 862-874. https://doi.org/10.1111/sms.12510

Laursen, B. P., \& Hoff, E. (2006). Person-centered and variable-centered approaches to longitudinal data. Merrill-Palmer Quarterly, 52(3), 377-389. https://doi.org/10.1353/mpq.2006.0029

Lazarus, R. S. (2000). How emotions influence performance in competitive sports. Sport Psychologist, 14(3), 229-252. https://doi.org/10.1123/tsp.14.3.229 
Louvet, B., Gaudreau, P., Menaut, A., Genty, J., \& Deneuve, P. (2009). Revisiting the changing and stable properties of coping utilization using latent class growth analysis: A longitudinal investigation with soccer referees. Psychology of Sport and Exercise, 10(1), 124-135. https://doi.org/10.1016/j.psychsport.2008.02.002

Lundqvist, C., \& Kenttä, G. (2010). Positive emotions are not simply the absence of negative ones: Development and validation of the Emotional Recovery Questionnaire (EmRecQ). The Sport Psychologist, 24, 468-488. http://dx.doi.org/10.1123/tsp.24.4.468.

Lyubomirsky, S., King, L., \& Diener, E. (2005). The benefits of frequent positive affect: Does happiness lead to success? Psychological bulletin, 131(6), 803. https://doi.org/10.1037/0033-2909.131.6.803

Martinent, G., Campo, M., \& Ferrand, C. (2012). A descriptive study of emotional process during competition: Nature, frequency, direction, duration and co-occurrence of discrete emotions. Psychology of Sport and Exercise, 13(2), 142-151. https://doi.org/10.1016/j.psychsport.2011.10.006

Martinent, G., Gareau, A., Lienhart, N., Nicaise, V., \& Guillet-Descas, E. (2018). Emotion profiles and their motivational antecedents among adolescent athletes in intensive training settings. Psychology of Sport and Exercise. https://doi.org/10.1016/j.psychsport.2018.01.001

Martinent, G., Ledos, S., Ferrand, C., Campo, M., \& Nicolas, M. (2015). Athletes' regulation of emotions experienced during competition: A naturalistic video-assisted study. Sport, Exercise, and Performance Psychology, 4(3), 188-205. https://doi.org/10.1037/spy0000037 
Martinent, G., Louvet, B., \& Decret, J.-C. (2016). Longitudinal trajectories of athlete burnout among young table tennis players: A 3-wave study. Journal of Sport and Health Science. https://doi.org/10.1016/j.jshs.2016.09.003

Mayer, J. D. (2009). Personal intelligence expressed: A theoretical analysis. Review of General Psychology, 13(1), 46-58. https://doi.org/10.1037/a0014229

McCarthy, P. J. (2011). Positive emotion in sport performance: current status and future directions. International Review of Sport and Exercise Psychology, 4(1), 50-69. https://doi.org/10.1080/1750984X.2011.560955

Mikolajczak, M. (2009). Going beyond the ability-trait debate: The three-level model of emotional intelligence an unifying view. e-Journal of Applied Psychology, 5, 25-31.

Nelis, D., Quoidbach, J., Mikolajczak, M., \& Hansenne, M. (2009). Increasing emotional intelligence: (How) is it possible? Personality and Individual Differences, 47, 36-41. doi:10.1016/j.paid.2009.01.046

Nicholls, A. R., Holt, N. L., Polman, R. C., \& Bloomfield, J. (2006). Stressors, coping, and coping effectiveness among professional rugby union players. The Sport Psychologist, 20(3), 314-329. https://doi.org/10.1123/tsp.20.3.314

Nylund-Gibson, K., Grimm, R., Quirk, M., \& Furlong, M. (2014). A latent transition mixture model using the three-step specification. Structural Equation Modeling: A Multidisciplinary Journal, 21(3), 439-454. https://doi.org/10.1080/10705511.2014.915375

Riemer, H. A., \& Chelladurai, P. (1998). Development of the athlete satisfaction questionnaire (ASQ). Journal of Sport and Exercise Psychology, 20(2), 127-156. https://doi.org/10.1123/jsep.20.2.127 
653 Rindfleisch, A., Malter, A. J., Ganesan, S., \& Moorman, C. (2008). Cross-sectional versus 654 longitudinal survey research: Concepts, findings, and guidelines. Journal of Marketing 655 Research, 45(3), 261-279. https://doi.org/10.1509/jmkr.45.3.261

656 Schutte, N. S., Malouff, J. M., Simunek, M., McKenley, J., \& Hollander, S. (2002).

657 Characteristic emotional intelligence and emotional well-being. Cognition \& Emotion, 658 16(6), 769-785. https://doi.org/10.1080/02699930143000482

659 Wagstaff, C. R. (2014). Emotion regulation and sport performance. Journal of Sport and 660 Exercise Psychology, 36(4), 401-412. https://doi.org/10.1123/jsep.2013-0257 
Table 1. Fit Indices of Latent Classe Growth Analysis Models With $1-6$ Classes for Emotions.

\begin{tabular}{lcccccc}
\hline & 1 class & 2 class & 3 class & 4 class & 5 class & 6 class \\
\hline Love & & & & & & \\
Log likelihood & -922.08 & -867.34 & -844.58 & $\mathbf{- 8 2 4 . 8 7}$ & -805.81 & -823.22 \\
AIC & 1856.16 & 1754.67 & 1717.15 & $\mathbf{1 6 8 5 . 7 4}$ & 1655.62 & 1698.44 \\
BIC & 1879.65 & 1793.83 & 17771.98 & $\mathbf{1 7 5 6 . 2 3}$ & 1741.77 & 1800.27 \\
ABIC & 1860.62 & 1762.11 & 1727.56 & $\mathbf{1 6 9 9 . 1 2}$ & 1672 & 1717.78 \\
LRT & N/A $^{\mathrm{a}}$ & $109.49^{*}$ & 45.52 & $\mathbf{3 9 . 4 2 *}$ & $38.12^{*}$ & 17.65 \\
Anxiety & & & & & & \\
Log likelihood & -1225.08 & $\mathbf{- 1 1 4 2 . 3 9}$ & -1121.97 & -1114.46 & -1109.28 & -1101.03 \\
AIC & 2462.15 & $\mathbf{2 3 0 4 . 7 8}$ & 2271.95 & 2264.92 & 2262.56 & 2254.05 \\
BIC & 2485.65 & $\mathbf{2 3 4 3 . 9 4}$ & 2326.77 & 2335.41 & 2348.72 & 2355.87 \\
ABIC & 2466.61 & $\mathbf{2 3 1 2 . 2 2}$ & 2282.36 & 2278.30 & 2278.92 & 2273.38 \\
LRT & N/A $^{\mathrm{a}}$ & $\mathbf{1 6 5 . 3 7 *}$ & 40.83 & 15.03 & 10.35 & 16.51 \\
Anger & & & & & & \\
Log likelihood & -1135.29 & -1038.81 & $\mathbf{- 1 0 1 4 . 5 4}$ & -991.19 & -997.61 & -965.34 \\
AIC & 2282.57 & 2097.63 & $\mathbf{2 0 5 7 . 0 9}$ & 2018.39 & 1999.21 & 1982.34 \\
BIC & 2306.07 & 2136.79 & $\mathbf{2 1 1 1 . 9 2}$ & 2088.88 & 2085.37 & 2084.17 \\
ABIC & 2287.04 & 2105.06 & $\mathbf{2 0 6 7 . 5 0}$ & 2031.77 & 2015.57 & 2001.68 \\
LRT & N/A $^{\mathrm{a}}$ & $192.95 *$ & $\mathbf{4 8 . 5 4 *}$ & $46.70 *$ & 27.17 & 24.87
\end{tabular}

Confidence (without including quadratic parameters)

Log likelihood $\quad-1202.70 \quad-1127.51 \quad-1105.03$

$\begin{array}{llll}\text { AIC } & 2415.40 & 2271.01 & 2232.06\end{array}$

$\begin{array}{llll}\mathrm{BIC} & 2434.98 & 2302.34 & 2275.14\end{array}$

ABIC $\quad 2419.11 \quad 2276.96 \quad 2240.24$

$\mathbf{- 1 0 8 3 . 9 4}-1070.04 \quad-1069.88$

2195.89 $2174.08 \quad 2179.77$

2250.71 $2240.66 \quad 2258.09$

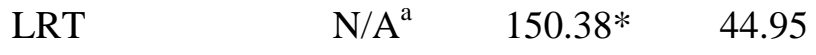

2206.30 $2186.72 \quad 2194.64$

Harmony (without including quadratic parameters)

\begin{tabular}{lcccccc} 
Log likelihood & -1079.97 & -1011.62 & $\mathbf{- 9 8 9 . 4 6}$ & -979.98 & -971.19 & -963.82 \\
AIC & 2169.95 & 2039.24 & $\mathbf{2 0 0 0 . 9 1}$ & 1987.95 & 1976.37 & 1867.64 \\
BIC & 2189.83 & 2070.57 & $\mathbf{2 0 4 3 . 9 9}$ & 2042.78 & 2042.95 & 2045.96 \\
ABIC & 2173.66 & 2045.19 & $\mathbf{2 0 0 9 . 0 9}$ & 4998.36 & 1989.01 & 1982.51 \\
LRT & N/A $^{\mathrm{a}}$ & $136.71^{*}$ & $\mathbf{4 4 . 3 3 *}$ & $18.96^{*}$ & 17.58 & 14.73 \\
Happiness & & & & & & \\
Log likelihood & -986.786 & -926.63 & $\mathbf{- 9 1 0 . 5 1}$ & -902.20 & -889.95 & -880.87 \\
AIC & 1985.57 & 1873.25 & $\mathbf{1 8 4 9 . 0 1}$ & 1840.41 & 1823.90 & 1813.74 \\
BIC & 2009.07 & 1912.42 & $\mathbf{1 9 0 3 . 8 4}$ & 1910.90 & 1910.06 & 1915.56 \\
ABIC & 1990.03 & 1880.69 & $\mathbf{1 8 5 9 . 4 2}$ & 1853.79 & 1840.26 & 1833.07 \\
LRT & N/A $^{\mathrm{a}}$ & $120.32^{*}$ & $\mathbf{3 2 . 2 4} *$ & 16.61 & $24.50 *$ & 19.52 \\
Sadness & & & & & & \\
Log likelihood & -1142.90 & -1017.79 & $\mathbf{- 9 7 5 . 0 3}$ & -953.47 & -930.46 & -912.75 \\
AIC & 2297.81 & 2055.57 & $\mathbf{1 9 7 8 . 0 6}$ & 1942.95 & 1904.91 & 1877.49 \\
BIC & 2321.30 & 2094.74 & $\mathbf{2 0 3 2 . 8 9}$ & 2013.44 & 1991.07 & 1979.31 \\
ABIC & 2302.27 & 2063.01 & $\mathbf{1 9 8 8 . 4 7}$ & 1956.33 & 1921.27 & 1896.82 \\
LRT & N/A & $250.23^{\mathrm{a}}$ & $\mathbf{8 5 . 5 1 *}$ & 43.12 & 46.04 & 35.42 \\
Vitality (without & including quadratic parameters) & & & \\
Log likelihood & -961.69 & -900.40 & $\mathbf{- 8 8 0 . 8 0}$ & -868.54 & -863.19 & -854.80 \\
AIC & 1933.39 & 1816.80 & $\mathbf{1 7 8 3 . 6 1}$ & 1765.09 & 1760.37 & 1749.59 \\
BIC & 1952.97 & 1848.13 & $\mathbf{1 8 2 6 . 6 8}$ & 1819.92 & 1826.95 & 1827.92 \\
ABIC & 1937.11 & 1822.75 & $\mathbf{1 7 9 1 . 7 8}$ & 1775.50 & 1773.01 & 1764.46 \\
LRT & N/A $^{\mathrm{a}}$ & $122.59^{*}$ & $\mathbf{3 9 . 1 9 *}$ & 24.52 & 12.97 & 8.89 \\
\hline Bo & & &
\end{tabular}

Note: Bold entries reflect selected model. ${ }^{a}$ Not available. ${ }^{*} p<0.05$. AIC $=$ Akaike Information Criterion; $\mathrm{BIC}=$ Bayesian Information Criterion; $\mathrm{ABIC}=$ Adjusted BIC; LRT = Lo, Mendell, and Rubin Likelihood Ratio Test. 
Table 2. Longitudinal Trajectories of Emotions across the 3 Waves.

\begin{tabular}{|c|c|c|c|c|c|c|c|}
\hline & \multirow[b]{2}{*}{$N$} & \multicolumn{2}{|l|}{ Intercept } & \multicolumn{2}{|l|}{ Linear } & \multicolumn{2}{|l|}{ Quadratic } \\
\hline & & Estimate (SE) & $p$ & Estimate (SE) & $p$ & Estimate (SE) & $p$ \\
\hline \multicolumn{8}{|l|}{ Love } \\
\hline High and stable & 181 & $3.86(.08)$ & .00 & $-0.08(.11)$ & .49 & $0.06(.05)$ & .21 \\
\hline Very high and unstable & 102 & $4.47(.05)$ & .00 & $-0.15(.12)$ & .20 & $0.17(.06)$ & .00 \\
\hline High and unstable & 33 & $3.77(.14)$ & .00 & $0.39(.22)$ & .08 & $-0.37(.10)$ & .00 \\
\hline Moderate and stable & 9 & $3.46(.32)$ & .00 & $-0.67(.78)$ & .39 & $-0.02(.33)$ & .96 \\
\hline \multicolumn{8}{|l|}{ Anxiety } \\
\hline Moderate and unstable & 130 & $3.08(.18)$ & .00 & $0.42(.22)$ & .06 & $-0.19(.11)$ & .09 \\
\hline Low and stable & 195 & $1.88(.09)$ & .00 & $0.12(.17)$ & .49 & $-0.09(.09)$ & .28 \\
\hline \multicolumn{8}{|l|}{ Anger } \\
\hline Moderate and stable & 93 & $2.60(.17)$ & .00 & $-0.20(.38)$ & .59 & $0.10(.16)$ & .55 \\
\hline High and decreasing & 22 & $3.87(.21)$ & .00 & $-0.88(.49)$ & .07 & $0.27(.25)$ & .27 \\
\hline Low and unstable & 210 & $1.37(.04)$ & .00 & $0.47(.13)$ & .00 & $-0.18(.06)$ & .01 \\
\hline \multicolumn{8}{|l|}{ Confidence } \\
\hline High and decreasing & 173 & $3.62(.10)$ & .00 & $-0.93(.05)$ & .08 & & \\
\hline Low and increasing & 52 & $2.24(.15)$ & .00 & $0.19(.10)$ & .05 & & \\
\hline High and increasing & 89 & $4.37(.09)$ & .00 & $0.15(.06)$ & .02 & & \\
\hline Moderate and increasing & 11 & $2.61(.43)$ & .00 & $1.06(.10)$ & .00 & & \\
\hline \multicolumn{8}{|l|}{ Harmony } \\
\hline High and stable & 145 & $4.30(.08)$ & .00 & $-0.01(.05)$ & .85 & & \\
\hline Moderate and stable & 166 & $3.29(.09)$ & .00 & $0.05(.05)$ & .32 & & \\
\hline Low and stable & 14 & $1.88(.17)$ & .00 & $0.20(.13)$ & .13 & & \\
\hline \multicolumn{8}{|l|}{ Happiness } \\
\hline Very high and decreasing & 101 & $4.69(.06)$ & .00 & $-0.43(.17)$ & .01 & $0.13(.08)$ & .13 \\
\hline Moderate and increasing & 40 & $2.66(.13)$ & .00 & $0.64(.30)$ & .03 & $-0.15(.15)$ & .30 \\
\hline High and stable & 180 & $3.72(.06)$ & .00 & $-0.13(.11)$ & .26 & $0.07(.06)$ & .25 \\
\hline \multicolumn{8}{|l|}{ Sadness } \\
\hline Low and stable & 241 & $1.33(.04)$ & .00 & $0.11(.14)$ & .44 & $-0.03(.07)$ & .72 \\
\hline High and unstable & 33 & $3.80(.14)$ & .00 & $-1.80(.35)$ & .00 & $0.68(.17)$ & .00 \\
\hline Low and unstable & 51 & $1.77(.15)$ & .00 & $2.32(.51)$ & .00 & $-0.93(.27)$ & .00 \\
\hline \multicolumn{8}{|l|}{ Vitality } \\
\hline High and stable & 133 & $4.66(.05)$ & .00 & $-0.06(.07)$ & .44 & & \\
\hline Low and increasing & 27 & $2.75(.24)$ & .00 & $0.40(.20)$ & .05 & & \\
\hline Moderate and decreasing & 165 & $3.88(.10)$ & .00 & $-0.11(.06)$ & .07 & & \\
\hline
\end{tabular}


Table 3. Association of Time 1 (T1) Emotional Regulation and Emotional Intelligence with Trajectories of Emotions.

\begin{tabular}{|c|c|c|c|}
\hline Model & Estimate & Odds ratio & $P$ \\
\hline \multicolumn{4}{|l|}{ Love } \\
\hline \multicolumn{4}{|l|}{ Trajectory: High and stable } \\
\hline T1 Reappraisal & -.12 & .89 & .51 \\
\hline T1 Suppression & .23 & 1.26 & .14 \\
\hline T1 Emotional Intelligence & -1.11 & .33 & .02 \\
\hline \multicolumn{4}{|l|}{ Trajectory: High and unstable } \\
\hline T1 Reappraisal & -.09 & .92 & .65 \\
\hline T1 Suppression & .15 & 1.16 & .39 \\
\hline T1 Emotional Intelligence & -1.28 & .28 & .02 \\
\hline \multicolumn{4}{|l|}{ Trajectory: Moderate and stable } \\
\hline T1 Reappraisal & .16 & 1.17 & .58 \\
\hline T1 Suppression & .26 & 1.30 & .57 \\
\hline T1 Emotional Intelligence & -1.47 & .23 & .10 \\
\hline \multicolumn{4}{|c|}{ Trajectory: Very high and unstable (constant) } \\
\hline \multicolumn{4}{|l|}{ Anxiety } \\
\hline \multicolumn{4}{|l|}{ Trajectory: Low and stable } \\
\hline T1 Reappraisal & .17 & 1.18 & .23 \\
\hline T1 Suppression & -.17 & .84 & .18 \\
\hline T1 Emotional Intelligence & .10 & 1.10 & .74 \\
\hline \multicolumn{4}{|c|}{ Trajectory: Moderate and unstable (constant) } \\
\hline \multicolumn{4}{|l|}{ Anger } \\
\hline \multicolumn{4}{|l|}{ Trajectory: Moderate and stable } \\
\hline T1 Reappraisal & .32 & 1.38 & .17 \\
\hline T1 Suppression & -.12 & .89 & .64 \\
\hline T1 Emotional Intelligence & -.64 & .53 & .17 \\
\hline \multicolumn{4}{|l|}{ Trajectory: Low and unstable } \\
\hline T1 Reappraisal & .48 & 1.61 & .03 \\
\hline T1 Suppression & -.33 & .72 & .11 \\
\hline T1 Emotional Intelligence & -.46 & .63 & .29 \\
\hline \multicolumn{4}{|c|}{ Trajectory: High and decreasing (constant) } \\
\hline \multicolumn{4}{|l|}{ Confidence } \\
\hline \multicolumn{4}{|l|}{ Trajectory: High and decreasing } \\
\hline T1 Reappraisal & -.16 & .85 & .39 \\
\hline T1 Suppression & -.03 & .97 & .88 \\
\hline T1 Emotional Intelligence & -1.35 & .26 & .02 \\
\hline \multicolumn{4}{|l|}{ Trajectory: Low and increasing } \\
\hline T1 Reappraisal & -.45 & .64 & .04 \\
\hline T1 Suppression & .13 & 1.14 & .49 \\
\hline T1 Emotional Intelligence & -.96 & .38 & .06 \\
\hline \multicolumn{4}{|c|}{ Trajectory: Moderate and increasing } \\
\hline T1 Reappraisal & .08 & 1.08 & .94 \\
\hline T1 Suppression & .27 & 1.31 & .62 \\
\hline T1 Emotional Intelligence & -1.80 & .17 & .13 \\
\hline \multicolumn{4}{|c|}{ Trajectory: High and increasing (constant) } \\
\hline Harmony & & & \\
\hline
\end{tabular}


Trajectory: Moderate and stable

T1 Reappraisal

$-.49 \quad .61$

.02

T1 Suppression

$.27 \quad 1.30$

.16

T1 Emotional Intelligence

$-1.96$

.14

.00

Trajectory: Low and stable

T1 Reappraisal

$\begin{array}{ll}-.80 & .45\end{array}$

.00

T1 Suppression

$\begin{array}{ll}.46 & 1.59\end{array}$

.08

T1 Emotional Intelligence

$-0.71$

.49

.22

Trajectory: High and stable (constant)

Happiness

Trajectory: Moderate and increasing

T1 Reappraisal

$-1.00$

.37

.00

T1 Suppression

.55

1.73

.01

T1 Emotional Intelligence

$-2.08$

.13

.00

Trajectory: High and stable

T1 Reappraisal

$\begin{array}{ll}-.26 & .77\end{array}$

.10

T1 Suppression

$.34 \quad 1.41$

.04

T1 Emotional Intelligence

$-1.73$

.18

.00

Trajectory: Very high and decreasing (constant)

Sadness

Trajectory: Low and stable

T1 Reappraisal

$\begin{array}{ll}.40 & 1.49\end{array}$

.02

T1 Suppression

$-.36$

.70

.02

T1 Emotional Intelligence

$-.30 \quad .74$

.41

Trajectory: Low and unstable

T1 Reappraisal

$\begin{array}{lll}.52 & 1.68 & \mathbf{0 3}\end{array}$

T1 Suppression

$\begin{array}{ll}-.22 & .80\end{array}$

.36

T1 Emotional Intelligence

$-.18$

.84

.69

Vitality

Trajectory: High and unstable (constant)

Trajectory: Low and increasing

T1 Reappraisal

T1 Suppression

T1 Emotional Intelligence

$-.64 \quad .53$

.01

$.33 \quad 1.39$

.16

$-1.35$

.26

.01

Trajectory: Moderate and decreasing

T1 Reappraisal

T1 Suppression

$\begin{array}{ll}-.43 & .65\end{array}$

.01

.32

1.38

T1 Emotional Intelligence

$-.65$

.52

.04

.11 